\title{
Acute unilateral foot drop as a result of direct blunt trauma to the peroneal nerve in a professional mixed marital arts bout: A case report
}

\author{
N K Sethi' ${ }^{1}, M D$, V Khabie ${ }^{2}$, MD \\ ${ }^{1}$ Department of Neurology, New York-Presbyterian Hospital, Weill Cornell Medical Center, New York, NY, USA \\ ${ }^{2}$ Department of Orthopedic Surgery, NYU-Hospital for Joint Diseases Orthopedic Institute, New York, NY, USA
}

Corresponding author: N K Sethi (sethinitinmd@hotmail.com)

As a result of its superficial location, the peroneal nerve is prone to compression injuries. This is a case report of an acute unilateral foot drop which occurred during a professional mixed martial arts (MMA) contest, specifically as a result of direct blunt trauma to the left peroneal nerve, without an accompanying fracture of the fibula.

Keywords: foot extensor weakness, gait abnormality, contact sports, mixed martial arts

S Afr J Sports Med 2017; 29:1. DOI: 10.17159/2078-516X/2017/v29i0a3561

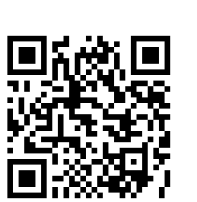

\section{Case report}

During the course of a professional MMA contest, a 22-year-old fighter was noted to suddenly have a "floppy" left foot which caused him difficulty in maintaining his balance during the fight. A medical timeout was called by the referee and the ringside physician entered the cage to evaluate the fighter. The fighter had a complete left foot drop and a step-off was palpable in the left fibula. However, the fighter denied feeling any pain when palpated along the length of the fibula. The fight was stopped on the advice of the ringside physician as a concern for acute peroneal nerve palsy, associated with a possible fibula fracture, was a possibility. Examination in the post-fight area revealed weak ankle dorsiflexion (Medical Research Council [MRC] grade 0/5) and big toe extension (MRC grade 0/5). Foot eversion was 0/5, inversion was preserved. Ankle and toe plantar flexion, knee flexion, as well as hip abduction, extension, and internal rotation, were normal. Weight bearing did not elicit any pain from the fighter. The Achilles tendon and patellar reflexes and sensation were not checked. The fighter was immediately transported to the nearest Level I trauma centre via ambulance where radiographs revealed no fracture of the fibula. He declined a stretcher and was able to walk to the ambulance aided by his coach and corner staff.

\section{Discussion}

Foot drop is defined as weakness of the foot and ankle dorsiflexion due to debility of the anterior tibialis, extensor halluces longus and extensor digitorum longus muscles. Foot drop can be found as a result of central and peripheral causes. It can occur if there is a disruption anywhere along the neural pathway, from the parasagittal cortical motor neurons to the spinal cord's upper motor neurons and the peripheral spinal motor neurons, ending with the peroneal nerve. ${ }^{[1]}$

A sciatic and peroneal nerve compromise can occur due to numerous traumatic and non-traumatic causes. Traumatic causes may occur in isolation or in association with musculoskeletal injuries involving the hip, knee, fibular head, tibia and the ankle. While acetabular and femur fractures, as well as posterior hip dislocations may compromise the sciatic nerve, the common peroneal nerve may be injured in fractures of the tibia and proximal fibula, and in knee dislocations. In lower extremity injuries, peroneal neuropathy may accompany ligamentous knee injury. Ligamentous and bony injuries of the ankle may also result in peroneal neuropathy. ${ }^{[2]}$

The peroneal nerve, due to its superficial course in the leg, is prone to compression as it winds closely around the fibula head and usually presents with a sub-acute and incomplete foot drop. The authors' presentation of an acute foot drop occurring during a MMA fight has not been previously reported. There was no accompanying fracture of the fibula leading to the authors' hypothesis that in this fighter the cause of the acute foot drop was a blunt trauma, most likely a kick to the lateral aspect of the leg. This resulted in an acute compression neurapraxia, although a more serious injury to the nerve could not be ruled out in the acute setting. A nerve conduction study carried out two to three weeks post injury, especially if recovery is poor or delayed, may aid in prognostication.

Author contributions: NKS conceived, drafted and revised the manuscript, VK reviewed and revised the manuscript.

Study funding: No targeted funding reported.

Disclosures: NKS serves as Associate Editor, The Eastern Journal of Medicine. VK reports no relevant disclosures.

Data sharing statement: The authors have no additional data to share.

\section{References}

1. Kertmen H, Gürer B, Yimaz ER, et al. Acute bilateral isolated foot drop: Report of two cases. Asian J Neurosurg 2015;10:123-125. [doi: 10.4103/1793-5482.144596]

2. Baima J, Krivickas L. Evaluation and treatment of peroneal neuropathy. Curr Rev Musculoskelet Med 2008;1:147-153. [doi: 10.1007/s12178-008-9023-6] 